\title{
The set of ratios of derangements to permutations in digraphs is dense in $[0,1 / 2]$
}

\author{
Bethany Austhof \\ Department of Mathematics, Statistics, and Computer Science \\ University of Illinois Chicago \\ Chicago, IL, U.S.A. \\ baust2@uic.edu \\ Patrick Bennett* \\ Department of Mathematics \\ Western Michigan University \\ Kalamazoo, MI, U.S.A. \\ patrick. bennett@wmich.edu \\ Nick Christo \\ Department of Mathematics, Statistics, and Computer Science \\ University of Illinois Chicago \\ Chicago, IL, U.S.A. \\ nchrist5@uic.edu
}

Submitted: Mar 9, 2021; Accepted: Nov 11, 2021; Published: Jan 28, 2022

(C) The authors. Released under the CC BY-ND license (International 4.0).

\begin{abstract}
A permutation in a digraph $G=(V, E)$ is a bijection $f: V \rightarrow V$ such that for all $v \in V$ we either have that $f$ fixes $v$ or $(v, f(v)) \in E$. A derangement in $G$ is a permutation that does not fix any vertex. Bucic, Devlin, Hendon, Horne and Lund proved that in any digraph, the ratio of derangements to permutations is at most $1 / 2$. Answering a question posed by Bucic, Devlin, Hendon, Horne and Lund, we show that the set of possible ratios of derangements to permutations in digraphs is dense in the interval $[0,1 / 2]$.
\end{abstract}

Mathematics Subject Classifications: 05A05, 05C 80

${ }^{*}$ Research supported in part by Simons Foundation Grant \#426894. 


\section{Introduction}

A permutation in a digraph (with no loops) $G=(V, E)$ is a bijection $f: V \rightarrow V$ such that for all $v \in V$ we either have that $f$ fixes $v$ or $(v, f(v)) \in E$. A derangement in $G$ is a permutation that does not fix any vertex. We define the parameter $(d / p)_{G}$ to be the ratio of derangements to permutations in $G$. As an aside, it is worth noting that if $A$ is the adjacency matrix of the graph $G$, then the ratio we are studying can be written

$$
(d / p)_{G}=\frac{\operatorname{per}(A)}{\operatorname{per}\left(A+I_{n}\right)}
$$

where $\operatorname{per}(\cdot)$ refers to the permanent of a matrix and $I_{n}$ is the $n \times n$ identity matrix.

Bucic, Devlin, Hendon, Horne and Lund [1] showed that $(d / p)_{G} \leqslant 1 / 2$ for all digraphs $G$, with equality if and only if $G$ is a directed cycle. They also gave a construction (the blow-up of a directed cycle) that can achieve a ratio arbitrarily close to but not equal to $1 / 2$. Let $S=\left\{(d / p)_{G}: G\right.$ is a digraph $\}$ be the set of values arising as a ratio $(d / p)$. In [1] they analyzed the ratio $(d / p)_{G}$ for the random graph $G=G(n, m)$, and as a corollary of this analysis they showed that $S$ is dense in $[0,1 / e]$. This corollary follows from two facts: $(d / p)_{G}$ is concentrated around its mean, and by choosing a suitable value of $m$ one can make the expected ratio $(d / p)_{G}$ close to any given value in $[0,1 / e]$. At the end of the paper [1] they ask whether $S$ is dense in $[0,1 / 2]$. Our main theorem, below, answers this question in the positive.

Theorem 1. The set of possible ratios of derangements to permutations in digraphs is dense in $[0,1 / 2]$.

The construction we use, described in more detail later, is a random subgraph of the blow-up of a directed cycle. The main part of the proof is an application of the second moment method (see [2], for example, for an introduction to the method) to show that the number of derangements and permutations are concentrated around their expectations.

\section{Proof of Theorem 1}

\subsection{Outline}

First we outline the proof. Suppose we are given a fixed real number $r \in[0,1 / 2]$. We will show that there exists a sequence of digraphs $G_{k}$ such that the ratio of derangements to permutations in $G_{k}$ is $r+o(1)$ as $k \rightarrow \infty$ (which proves Theorem 1 ). If $r=0$ or $1 / 2$ this is trivial. Indeed, for $r=0$ observe that a digraph with one vertex and no edges has no derangements and one permutation, and for $r=1 / 2$ observe that any directed cycle has one derangement and two permutations. So we assume $0<r<1 / 2$.

Our construction is as follows. As defined in [1], let the digraph $D_{k, \ell}$ where $k \geqslant 1$ and $\ell \geqslant 2$ have vertices $v_{i j}$ for $i \in[k]$ and $j \in[\ell]$ such that $\left(v_{i j}, v_{l m}\right) \in E\left(D_{k, \ell}\right)$ if and only if $m=j+1 \bmod l$. In other words, $D_{k, \ell}$ is the blow-up of a directed $\ell$-cycle where each vertex is expanded to a set of $k$ vertices. We let $V_{i}=\left\{v_{i j}: j \in[\ell]\right\}$. As was shown 
in [1], the number of derangements on $D_{k, \ell}$ is $(k !)^{\ell}$ and the number of permutations on $D_{k, \ell}$ is $\sum_{i=0}^{k}\left(\left(\begin{array}{c}k \\ i\end{array}\right)(k-i) !\right)^{\ell}$. Hence, $(d / p)_{k, \ell}=\left(\sum_{i=0}^{k}\left(\frac{1}{i !}\right)^{\ell}\right)^{-1}$ can be made arbitrarily close to $1 / 2$ by choosing $\ell$ large enough (even for large $k$ ). This construction yields a graph for which the ratio of derangements to permutations is arbitrarily close to $1 / 2$ but not exactly $1 / 2$. We will also use this construction, but we will randomly remove some edges. By taking a random subgraph we can "interpolate" between $D_{k, \ell}$ (a dense digraph whose ratio of derangements to permutations is close to $1 / 2$ ) and a sparse random digraph (whose ratio is 0 ).

In this paper all asymptotics are as $k \rightarrow \infty$. $\ell$ is treated as fixed. We use standard big-O, little-o and $\Omega$ notation. We write $x \sim y$ if $x=(1+o(1)) y$. All logarithms are base $e$.

\subsection{Proof details}

Let the random graph $G_{k, \ell}(m)$ be chosen uniformly from among all subgraphs of $D_{k, \ell}$ with $m$ edges. We will fix some $p, \ell$ and let $m=p k^{2} \ell$ (so $p$ is the probability that any particular edge of $D_{k, \ell}$ becomes an edge of $G_{k, \ell}$ ). Let the random variables $X, Y$ be the number of derangements and permutations in $G_{k, \ell}(m)$ respectively. Let $\mathcal{D}, \mathcal{P}$ be the collection of all possible derangements and permutations on $D_{k, \ell}(m)$.

\subsubsection{First moments of $X, Y$}

We have

$$
\begin{aligned}
\mathbb{E}[X] & =\sum_{D \in \mathcal{D}} \mathbb{P}\left[D \subseteq G_{k, \ell}\right]=(k !)^{\ell} \frac{\left(\begin{array}{c}
k^{2} \ell-k \ell \\
m-k \ell
\end{array}\right)}{\left(\begin{array}{c}
k^{2} \ell \\
m
\end{array}\right)} \\
& =(k !)^{\ell}\left(\frac{m}{k^{2} \ell}\right)^{k \ell} \exp \left\{\frac{k^{2} \ell^{2}}{2}\left(\frac{1}{k^{2} \ell}-\frac{1}{m}\right)+O\left(\frac{k^{3}}{m^{2}}+\frac{k}{m}\right)\right\} \\
& \sim(k !)^{\ell} p^{k \ell} \exp \left\{\frac{\ell}{2}\left(1-\frac{1}{p}\right)\right\},
\end{aligned}
$$

where on the second line we have used the following fact:

Fact 2 .

$$
\frac{\left(\begin{array}{l}
a-x \\
b-x
\end{array}\right)}{\left(\begin{array}{l}
a \\
b
\end{array}\right)}=\frac{(b)_{x}}{(a)_{x}}=\left(\frac{b}{a}\right)^{x} \exp \left\{\frac{x^{2}}{2}\left(\frac{1}{a}-\frac{1}{b}\right)+O\left(\frac{x^{3}}{b^{2}}+\frac{x}{b}\right)\right\} .
$$

For completeness we include the proof although it is well-known.

Proof.

$$
\frac{(b)_{x}}{(a)_{x}}=\left(\frac{b}{a}\right)^{x} \cdot \frac{1\left(1-\frac{1}{b}\right)\left(1-\frac{2}{b}\right) \cdots\left(1-\frac{x-1}{b}\right)}{1\left(1-\frac{1}{a}\right)\left(1-\frac{2}{a}\right) \cdots\left(1-\frac{x-1}{a}\right)}
$$




$$
\begin{aligned}
& =\left(\frac{b}{a}\right)^{x} \cdot \exp \left\{\sum_{i=0}^{x-1}\left[\ln \left(1-\frac{i}{b}\right)-\ln \left(1-\frac{i}{a}\right)\right]\right\} \\
& =\left(\frac{b}{a}\right)^{x} \cdot \exp \left\{\sum_{i=0}^{x-1}\left[-\frac{i}{b}+\frac{i}{a}+O\left(\frac{i^{2}}{a^{2}}+\frac{i^{2}}{b^{2}}\right)\right]\right\} \\
& =\left(\frac{b}{a}\right)^{x} \cdot \exp \left\{\frac{x(x-1)}{2}\left(\frac{1}{a}-\frac{1}{b}\right)+O\left(\frac{x^{3}}{b^{2}}\right)\right\} \\
& =\left(\frac{b}{a}\right)^{x} \exp \left\{\frac{x^{2}}{2}\left(\frac{1}{a}-\frac{1}{b}\right)+O\left(\frac{x^{3}}{b^{2}}+\frac{x}{b}\right)\right\} .
\end{aligned}
$$

Before we calculate $\mathbb{E}[Y]$ we introduce a function $f_{\ell}(x)$. For any integer $\ell \geqslant 1$, let

$$
f_{\ell}(x):=\sum_{i=0}^{\infty} \frac{x^{i \ell}}{(i !)^{\ell}} .
$$

Note that the above power series for $f_{\ell}(x)$ converges for all $x$ and therefore in particular each $f_{\ell}$ is continuous in $x$. We have

$$
\begin{aligned}
\mathbb{E}[Y] & =\sum_{P \in \mathcal{P}} \mathbb{P}\left[P \subseteq G_{k, \ell}\right]=\sum_{i=0}^{k}\left(\left(\begin{array}{c}
k \\
i
\end{array}\right)(k-i) !\right)^{\ell} \frac{\left(\begin{array}{c}
k^{2} \ell-(k-i) \ell \\
m-(k-i) \ell
\end{array}\right)}{\left(\begin{array}{c}
k^{2} \ell \\
m
\end{array}\right)} \\
& =\sum_{i=0}^{k}\left(\frac{k !}{i !}\right)^{\ell}\left(\frac{m}{k^{2} \ell}\right)^{(k-i) \ell} \exp \left\{\frac{(k-i)^{2} \ell^{2}}{2}\left(\frac{1}{k^{2} \ell}-\frac{1}{m}\right)+O\left(\frac{k^{3}}{m^{2}}+\frac{k}{m}\right)\right\} \\
& =(k !)^{\ell} p^{k \ell} \sum_{i=0}^{k}\left(\frac{1}{i !}\right)^{\ell} p^{-i \ell} \exp \left\{\frac{\ell}{2}\left(1-\frac{1}{p}\right)+O\left(\frac{i+1}{k}\right)\right\} .
\end{aligned}
$$

We split the above sum into two ranges of $i$. Note that for $0 \leqslant i \leqslant \sqrt{k}$ we have $\exp \left\{O\left(\frac{i+1}{k}\right)\right\}=1+O\left(\frac{1}{\sqrt{k}}\right)$, while for $\sqrt{k} \leqslant i \leqslant k$ we have $\exp \left\{O\left(\frac{i+1}{k}\right)\right\}=$ $O(1)$. Thus line $(2.3)$ becomes

$$
\begin{aligned}
(k !)^{\ell} p^{k \ell}\left[\left(1+O\left(\frac{1}{\sqrt{k}}\right)\right) \exp \left\{\frac{\ell}{2}\left(1-\frac{1}{p}\right)\right\} \sum_{0 \leqslant i \leqslant \sqrt{k}}\left(\frac{1}{i !}\right)^{\ell} p^{-i \ell}\right. & \left.+O(1) \sum_{\sqrt{k}<i \leqslant k}\left(\frac{1}{i !}\right)^{\ell} p^{-i \ell}\right] .
\end{aligned}
$$

As $k \rightarrow \infty$ we have

$$
\sum_{0 \leqslant i \leqslant \sqrt{k}}\left(\frac{1}{i !}\right)^{\ell} p^{-i \ell} \rightarrow f_{\ell}(1 / p)
$$


and

$$
\sum_{\sqrt{k}<i \leqslant k}\left(\frac{1}{i !}\right)^{\ell} p^{-i \ell} \leqslant \sum_{i=\sqrt{k}}^{\infty}\left(\frac{1}{i !}\right)^{\ell} p^{-i \ell}=o(1)
$$

since the latter is the tail of a convergent series. Thus, returning to our estimate of $\mathbb{E}[Y]$ on line (2.4), we have

$$
\mathbb{E}[Y] \sim(k !)^{\ell} p^{k \ell} \exp \left\{\frac{\ell}{2}\left(1-\frac{1}{p}\right)\right\} f_{\ell}(1 / p) .
$$

\subsubsection{Choosing $p, \ell$}

Now that we know $\mathbb{E}[X], \mathbb{E}[Y]$ we will choose $p, \ell$ to make sure that the ratio of $\mathbb{E}[X]$ to $\mathbb{E}[Y]$ is close to $r$. Using lines (2.1) and (2.5) we have

$$
\frac{\mathbb{E}[X]}{\mathbb{E}[Y]} \sim \frac{1}{f_{\ell}\left(\frac{1}{p}\right)}
$$

so we would like to choose $\ell$ and $0<p<1$ so that $f_{\ell}(1 / p)=1 / r$. We have

$$
\lim _{x \rightarrow \infty} f_{\ell}(x)=\infty, \quad f_{\ell}(1)=\sum_{i=0}^{k}\left(\frac{1}{i !}\right)^{\ell}=1+1+\frac{1}{2^{\ell}}+\frac{1}{6^{\ell}}+\frac{1}{24^{\ell}}+\ldots
$$

Note that we can make $f_{\ell}(1)$ arbitrarily close to 2 by taking $\ell$ large. Indeed, we have $f_{\ell}(1) \geqslant 2$ and

$$
f_{\ell}(1)=2+\sum_{i \geqslant 2}\left(\frac{1}{i !}\right)^{\ell} \leqslant 2+\sum_{i \geqslant 2}\left(\frac{1}{2^{i-1}}\right)^{\ell}=2+\frac{1}{2^{\ell}-1} .
$$

Since $r<1 / 2$, we can choose $\ell$ so that $f_{\ell}(1)<1 / r$. Then by the intermediate value theorem there is some $x \in(1, \infty)$ such that $f_{\ell}(x)=1 / r$. We choose $p$ to be the value $1 / x$, so $0<p<1$ and $f_{\ell}(1 / p)=1 / r$. So we view $\ell$ and $p$ as constants determined entirely by $r$.

\subsubsection{Second moments of $X, Y$}

In this section we show that $\mathbb{E}\left[X^{2}\right] \sim \mathbb{E}[X]^{2}$ and $\mathbb{E}\left[Y^{2}\right] \sim \mathbb{E}[Y]^{2}$. This will complete the proof, since then by the second moment method we have that

$$
\frac{X}{Y} \sim \frac{\mathbb{E}[X]}{\mathbb{E}[Y]} \sim \frac{1}{f_{\ell}(1 / p)}=r
$$

with probability approaching 1 as $k$ goes to infinity.

To help us estimate $\mathbb{E}\left[X^{2}\right], \mathbb{E}\left[Y^{2}\right]$ we will find the function $h(a, b)$ (defined below) useful. Suppose we have some fixed matching $B$ of $b$ many edges in the graph $K_{a, a}$. Then 
by inclusion-exclusion the number of perfect matchings that do not have any edges from $B$ is

$$
h(a, b):=\sum_{w=0}^{b}(-1)^{w}\left(\begin{array}{c}
b \\
w
\end{array}\right)(a-w) ! .
$$

Note that we always have $h(a, b) \leqslant a$ !. We will now observe that, roughly speaking, $h(a, b) \approx \frac{a !}{e}$ whenever $b \approx a \rightarrow \infty$. More formally we have the following

Fact 3. Suppose $a-a^{1 / 10} \leqslant b \leqslant a$. Then we have

$$
h(a, b)=\left(1+O\left(a^{-4 / 5}\right)\right) \frac{a !}{e}
$$

as $a \rightarrow \infty$

Proof. We have

$$
h(a, b)=\sum_{0 \leqslant w \leqslant b}(-1)^{w}\left(\begin{array}{c}
b \\
w
\end{array}\right)(a-w) !=a ! \sum_{0 \leqslant w \leqslant b} \frac{(-1)^{w}}{w !} \frac{(b)_{w}}{(a)_{w}} .
$$

Now, for $0 \leqslant w \leqslant a^{1 / 10}$ we have by Fact 2 that

$$
\begin{aligned}
\frac{(b)_{w}}{(a)_{w}} & =\left(\frac{b}{a}\right)^{w} \exp \left\{\frac{w^{2}}{2}\left(\frac{1}{a}-\frac{1}{b}\right)+O\left(\frac{w^{3}}{b^{2}}+\frac{w}{b}\right)\right\} \\
& =\left(1+O\left(a^{-9 / 10}\right)\right)^{O\left(a^{1 / 10}\right)} \exp \left\{O\left(a^{-4 / 5}\right\}=1+O\left(a^{-4 / 5}\right) .\right.
\end{aligned}
$$

Meanwhile for $w \geqslant a^{1 / 10}$ we have that the corresponding term in line (2.6) has absolute value

$$
\frac{1}{w !} \frac{(b)_{w}}{(a)_{w}} \leqslant \frac{1}{\left(a^{1 / 10}\right) !}=\exp \left\{-\Omega\left(a^{1 / 10} \log a\right)\right\}
$$

by Stirling's approximation. Thus, the sum of all such terms in line (2.6) is at most

$$
b \exp \left\{-\Omega\left(a^{1 / 10} \log a\right)\right\}=O\left(a^{-4 / 5}\right)
$$

(this bound is quite comfortable). By the Alternating Series Test we have that

$$
\sum_{0 \leqslant w \leqslant a^{1 / 10}} \frac{(-1)^{w}}{w !}=\frac{1}{e}+O\left(\frac{1}{\left(a^{1 / 10}\right) !}\right)=\frac{1}{e}+O\left(a^{-4 / 5}\right) .
$$

Breaking up the sum for $h(a, b)$ we have

$$
\begin{aligned}
h(a, b) & =a !\left[\sum_{0 \leqslant w \leqslant a^{1 / 10}} \frac{(-1)^{w}}{w !} \frac{(b)_{w}}{(a)_{w}}+\sum_{a^{1 / 10}<w \leqslant b} \frac{(-1)^{w}}{w !} \frac{(b)_{w}}{(a)_{w}}\right] \\
& =a !\left[\left(1+O\left(a^{-4 / 5}\right)\right) \sum_{0 \leqslant w \leqslant a^{1 / 10}} \frac{(-1)^{w}}{w !}+O\left(a^{-4 / 5}\right)\right] \\
& =\left(1+O\left(a^{-4 / 5}\right)\right) \frac{a !}{e} .
\end{aligned}
$$


We find that

$$
\begin{aligned}
\mathbb{E}\left[X^{2}\right] & =\sum_{D, D^{\prime} \in \mathcal{D}} \mathbb{P}\left[D, D^{\prime} \subseteq G_{k, \ell}\right]=(k !)^{\ell} \sum_{D^{\prime} \in \mathcal{D}} \mathbb{P}\left[D_{0}, D^{\prime} \subseteq G_{k, \ell}\right] \\
& =(k !)^{\ell} \sum_{b=0}^{k \ell}\left[\frac{\left(\begin{array}{c}
k^{2} \ell-(2 k \ell-b) \\
m-(2 k \ell-b)
\end{array}\right)}{\left(\begin{array}{c}
k^{2} \ell \\
m
\end{array}\right)} \sum_{\vec{b} \in S_{b}} \prod_{c=1}^{\ell}\left(\begin{array}{c}
k \\
b_{c}
\end{array}\right) h\left(k-b_{c}, k-b_{c}\right)\right],
\end{aligned}
$$

where $D_{0}$ is a fixed derangement and in the inner sum, $S_{b}$ is the set of $\ell$-dimensional vectors $\vec{b}=\left(b_{1}, \ldots b_{\ell}\right)$ whose components are nonnegative integers summing to $b$.

By 2 , if $b \leqslant k^{1 / 10}$ then we have

$$
\begin{aligned}
\frac{\left(\begin{array}{c}
k^{2} \ell-(2 k \ell-b) \\
m-(2 k \ell-b)
\end{array}\right)}{\left(\begin{array}{c}
k^{2} \ell \\
m
\end{array}\right)} & =p^{2 k \ell-b} \exp \left\{\frac{(2 k \ell-b)^{2}}{2}\left(\frac{1}{k^{2} \ell}-\frac{1}{m}\right)+O\left(\frac{k^{3}}{m^{2}}+\frac{k}{m}\right)\right\} \\
& =\left(1+O\left(k^{-9 / 10}\right)\right) p^{2 k \ell-b} \exp \left\{2 \ell\left(1-\frac{1}{p}\right)\right\},
\end{aligned}
$$

and by Fact 3 we have $h\left(k-b_{c}, k-b_{c}\right)=\left(1+O\left(k^{-4 / 5}\right)\right) \frac{\left(k-b_{c}\right) !}{e}$. Therefore the term corresponding to $b$ in $(2.7)$ is

$$
\begin{aligned}
& \frac{\left(\begin{array}{c}
k^{2} \ell-(2 k \ell-b) \\
m-(2 k \ell-b)
\end{array}\right)}{\left(\begin{array}{c}
k^{2} \ell \\
m
\end{array}\right)} \sum_{\vec{b} \in S_{b}} \prod_{c=1}^{\ell}\left(\begin{array}{c}
k \\
b_{c}
\end{array}\right) h\left(k-b_{c}, k-b_{c}\right) \\
& =\left(1+O\left(k^{-4 / 5}\right)\right) p^{2 k \ell-b} \exp \left\{2 \ell\left(1-\frac{1}{p}\right)\right\} \sum_{\vec{b} \in S_{b}} \prod_{c=1}^{\ell}\left(\begin{array}{c}
k \\
b_{c}
\end{array}\right) \frac{\left(k-b_{c}\right) !}{e} \\
& =\left(1+O\left(k^{-4 / 5}\right)\right)(k !)^{\ell} p^{2 k \ell-b} \exp \left\{2 \ell\left(1-\frac{1}{p}\right)-\ell\right\} \sum_{\vec{b} \in S_{b}} \prod_{c=1}^{\ell} \frac{1}{b_{c} !} \\
& =\left(1+O\left(k^{-4 / 5}\right)\right)(k !)^{\ell} p^{2 k \ell-b} \exp \left\{\ell\left(1-\frac{2}{p}\right)\right\} \frac{\ell^{b}}{b !},
\end{aligned}
$$

where on the last line we used the multinomial formula. Meanwhile if $b \geqslant k^{1 / 10}$ then the term corresponding to $b$ in $(2.7)$ is

$$
\begin{aligned}
& \frac{\left(\begin{array}{c}
k^{2} \ell-(2 k \ell-b) \\
m-(2 k \ell-b)
\end{array}\right)}{\left(\begin{array}{c}
k^{2} \ell \\
m
\end{array}\right)} \sum_{\vec{b} \in S_{b}} \prod_{c=1}^{\ell}\left(\begin{array}{c}
k \\
b_{c}
\end{array}\right) h\left(k-b_{c}, k-b_{c}\right) \\
& \leqslant \sum_{\vec{b} \in S_{b}} \prod_{c=1}^{\ell}\left(\begin{array}{c}
k \\
b_{c}
\end{array}\right)\left(k-b_{c}\right) ! \\
& =(k !)^{\ell} \frac{\ell^{b}}{b !}
\end{aligned}
$$




$$
=(k !)^{\ell} \cdot \exp \left\{-\Omega\left(k^{1 / 10} \log k\right)\right\},
$$

and so the sum of all terms in $(2.7)$ with $b \geqslant k^{1 / 10}$ is at most

$$
k \ell \cdot(k !)^{\ell} \cdot \exp \left\{-\Omega\left(k^{1 / 10} \log k\right)\right\}=(k !)^{\ell} \cdot O\left(k^{-4 / 5}\right) .
$$

Therefore,

$$
\begin{aligned}
\mathbb{E}\left[X^{2}\right]=(k !)^{\ell} \sum_{b=0}^{k \ell}\left[\frac{\left(\begin{array}{c}
k^{2} \ell-(2 k \ell-b) \\
m-(2 k \ell-b)
\end{array}\right)}{\left(\begin{array}{c}
k^{2} \ell \\
m
\end{array}\right)} \sum_{\vec{b} \in S_{b}} \prod_{c=1}^{\ell}\left(\begin{array}{c}
k \\
b_{c}
\end{array}\right) h\left(k-b_{c}, k-b_{c}\right)\right] \\
=(k !)^{\ell}\left[\sum_{0 \leqslant b \leqslant k^{1 / 10}}\left(1+O\left(k^{-4 / 5}\right)\right)(k !)^{\ell} p^{2 k \ell-b} \exp \left\{\ell\left(1-\frac{2}{p}\right)\right\} \frac{\ell^{b}}{b !}\right. \\
\left.+(k !)^{\ell} \cdot O\left(k^{-4 / 5}\right)\right] \\
=\left(1+O\left(k^{-4 / 5}\right)\right)(k !)^{2 \ell} p^{2 k \ell} \exp \left\{\ell\left(1-\frac{2}{p}\right)\right\} \sum_{0 \leqslant b \leqslant k^{1 / 10}} p^{-b} \frac{\ell^{b}}{b !} \\
=\left(1+O\left(k^{-4 / 5}\right)\right)(k !)^{2 \ell} p^{2 k \ell} \exp \left\{\ell\left(1-\frac{2}{p}\right)\right\} \cdot\left(\exp \left\{\frac{\ell}{p}\right\}+O\left(k^{-4 / 5}\right)\right) \\
=\left(1+O\left(k^{-4 / 5}\right)\right)(k !)^{2 \ell} p^{2 k \ell} \exp \left\{\ell\left(1-\frac{1}{p}\right)\right\} \\
\sim \mathbb{E}[X]^{2} .
\end{aligned}
$$

For $\mathbb{E}\left[Y^{2}\right]$ we find an exact expression to be cumbersome, but the following upper bound will suffice. We claim (with justification below) that $\mathbb{E}\left[Y^{2}\right]$ is at most

$$
\sum_{\substack{0 \leqslant i, j \leqslant k \\
0 \leqslant b \leqslant k \ell \\
\vec{b} \in S_{b}}} \frac{\left(\begin{array}{c}
k^{2} \ell-(2 k \ell-(i+j) \ell-b) \\
m-(2 k \ell-(i+j) \ell-b)
\end{array}\right)}{\left(\begin{array}{c}
k^{2} \ell \\
m
\end{array}\right)}\left(\frac{k !}{i !}\right)^{\ell} \prod_{c=1}^{\ell}\left(\begin{array}{c}
k-i \\
b_{c}
\end{array}\right)\left(\begin{array}{c}
k-b_{c} \\
j
\end{array}\right) h\left(k-j-b_{c}, k-i-b_{c}-2 j\right) .
$$

The term corresponding to a tuple $(i, j, b, \vec{b})$ above is an upper bound on the contribution to $\mathbb{E}\left[Y^{2}\right]$ due to pairs of permutations $\left(P, P^{\prime}\right)$ such that $P$ fixes $i$ vertices per part, $P^{\prime}$ fixes $j$ vertices per part, and $P$ and $P^{\prime}$ share a total of $b$ edges where $b_{c}$ of the shared edges are between $V_{c}$ and part $V_{c+1}$. The first factor is the edge probability, and the next factor is the number of choices for $P$. The next factor is an upper bound on the number of choices for $P^{\prime}$. Indeed, we choose the edges of $P^{\prime}$ from $V_{c}$ to $V_{c+1}$ by first choosing $b_{c}$ edges of $P$ to be shared, then we choose $j$ vertices in $V_{c}$ to be fixed by $P^{\prime}$, and finally we choose a matching between the remaining vertices (the vertices of $V_{c} \cup V_{c+1}$ that are not fixed by $P^{\prime}$ and are not endpoints of the $b_{c}$ shared edges already chosen). This matching 
must avoid any edges of $P$, and the vertices to be matched induce at least $k-i-b_{c}-2 j$ edges of $P$, explaining the last factor above.

We will now estimate the significant terms in (2.8). Assume $i, j, b \leqslant k^{1 / 10}$. Then by Fact 2

$$
\begin{aligned}
\frac{\left(\begin{array}{c}
k^{2} \ell-(2 k \ell-(i+j) \ell-b) \\
m-(2 k \ell-(i+j) \ell-b)
\end{array}\right)}{\left(\begin{array}{c}
k^{2} \ell \\
m
\end{array}\right)} & =p^{2 k \ell-(i+j) \ell-b} \exp \left\{\frac{(2 k \ell-(i+j) \ell-b)^{2}}{2 k^{2} \ell}\left(1-\frac{1}{p}\right)+O\left(\frac{1}{k}\right)\right\} \\
& =p^{2 k \ell-(i+j) \ell-b} \exp \left\{2 \ell\left(1-\frac{1}{p}\right)+O\left(k^{-9 / 10}\right)\right\} .
\end{aligned}
$$

Next we estimate

$$
h\left(k-j-b_{c}, k-i-b_{c}-2 j\right)=\left(1+O\left(k^{-4 / 5}\right)\right) \frac{\left(k-j-b_{c}\right) !}{e}
$$

by Fact 3 . So the product in $(2.8)$ is

$$
\begin{aligned}
& \prod_{c=1}^{\ell}\left(\begin{array}{c}
k-i \\
b_{c}
\end{array}\right)\left(\begin{array}{c}
k-b_{c} \\
j
\end{array}\right) h\left(k-j-b_{c}, k-i-b_{c}-2 j\right) \\
& \quad=\left(1+O\left(k^{-4 / 5}\right)\right) \prod_{c=1}^{\ell} \frac{(k-i)_{b_{c}}}{b_{c} !} \frac{\left(k-b_{c}\right)_{j}}{j !} \frac{\left(k-j-b_{c}\right) !}{e} \\
& \quad \leqslant\left(1+O\left(k^{-4 / 5}\right)\right)\left(\frac{k !}{e j !}\right)^{\ell} \prod_{c=1}^{\ell} \frac{1}{b_{c} !}
\end{aligned}
$$

The sum of terms in (2.8) corresponding to small $i, j, b$ is at most

$$
\begin{aligned}
(1 & \left.+O\left(k^{-4 / 5}\right)\right) \sum_{\substack{0 \leqslant i, j, b \leqslant k^{1 / 10} \\
\vec{b} \in S_{b}}} p^{2 k \ell-(i+j) \ell-b} \exp \left\{2 \ell\left(1-\frac{1}{p}\right)\right\} \cdot\left(\frac{k !}{i !}\right)^{\ell}\left(\frac{k !}{e j !}\right)^{\ell} \prod_{c=1}^{\ell} \frac{1}{b_{c} !} \\
& =\left(1+O\left(k^{-4 / 5}\right)\right) \exp \left\{\ell\left(1-\frac{2}{p}\right)\right\} \sum_{0 \leqslant i, j, b \leqslant k^{1 / 10}} p^{2 k \ell-(i+j) \ell-b}\left(\frac{k !}{i !}\right)^{\ell}\left(\frac{k !}{j !}\right)^{\ell} \frac{\ell^{b}}{b !} \\
& \leqslant\left(1+O\left(k^{-4 / 5}\right)\right)(k !)^{2 \ell} p^{2 k \ell} \exp \left\{\ell\left(1-\frac{1}{p}\right)\right\} \sum_{0 \leqslant i, j, b} \frac{p^{-i \ell}}{(i !)^{\ell}} \cdot \frac{p^{-j \ell}}{(j !)^{\ell}} \cdot \frac{(\ell / p)^{b}}{b !} \\
& =\left(1+O\left(k^{-4 / 5}\right)\right)(k !)^{2 \ell} p^{2 k \ell} \exp \left\{\ell\left(1-\frac{1}{p}\right)\right\} f_{\ell}\left(\frac{1}{p}\right)^{2} \\
& \sim \mathbb{E}[Y]^{2},
\end{aligned}
$$

where on the second-to-last line we have used

$$
\sum_{0 \leqslant i} \frac{p^{-i \ell}}{(i !)^{\ell}}=f_{\ell}\left(\frac{1}{p}\right), \quad \sum_{0 \leqslant b} \frac{(\ell / p)^{b}}{b !}=\exp \left\{\frac{\ell}{p}\right\} .
$$


It remains to show that the sum of all other terms (i.e. terms where $i, j$, or $b$ is at least $\left.k^{1 / 10}\right)$ is negligible compared to $\mathbb{E}[Y]^{2}$, which is of order $(k !)^{2 \ell} p^{2 k \ell}$. Note that by Fact 2

$$
\begin{aligned}
\frac{\left(\begin{array}{c}
k^{2} \ell-(2 k \ell-(i+j) \ell-b) \\
m-(2 k \ell-(i+j) \ell-b)
\end{array}\right)}{\left(\begin{array}{c}
k^{2} \ell \\
m
\end{array}\right)} & =p^{2 k \ell-(i+j) \ell-b} \exp \left\{\frac{(2 k \ell-(i+j) \ell-b)^{2}}{2 k^{2} \ell}\left(1-\frac{1}{p}\right)+O\left(\frac{1}{k}\right)\right\} \\
& =O\left(p^{2 k \ell-(i+j) \ell-b}\right) .
\end{aligned}
$$

Thus, the sum (over $\vec{b}$ ) of terms corresponding to a fixed triple $(i, j, b)$ in line (2.8) big-O of

$$
\begin{gathered}
p^{2 k \ell-(i+j) \ell-b} \sum_{\vec{b} \in S_{b}}\left(\frac{k !}{i !}\right)^{\ell} \prod_{c=1}^{\ell}\left(\begin{array}{c}
k-i \\
b_{c}
\end{array}\right)\left(\begin{array}{c}
k-b_{c} \\
j
\end{array}\right)\left(k-j-b_{c}\right) ! \\
\leqslant p^{2 k \ell-(i+j) \ell-b}\left(\frac{k !}{i !}\right)^{\ell}\left(\frac{k !}{j !}\right)^{\ell} \sum_{\vec{b} \in S_{b}} \prod_{c=1}^{\ell} \frac{1}{b_{c} !} \\
=\left((k !)^{2 \ell} p^{2 k \ell}\right) \cdot\left(\frac{\ell^{b}}{p^{(i+j) \ell+b}(i !)^{\ell}(j !)^{\ell} b !}\right) .
\end{gathered}
$$

It is easy to see that if $i, j$ or $b$ is at least $k^{1 / 10}$ then the second factor above is $\exp \left\{-\Omega\left(k^{1 / 10} \log k\right)\right\}$. Since the number of triples $(i, j, b)$ is polynomial in $k$, the sum of all such terms (i.e. where $i, j$ or $b$ is at least $\left.k^{1 / 10}\right)$ is $o\left((k !)^{2 \ell} p^{2 k \ell}\right)$ which is a negligible contribution to $\mathbb{E}\left[Y^{2}\right]$. Therefore we have $\mathbb{E}\left[Y^{2}\right] \sim \mathbb{E}[Y]^{2}$.

\section{Remarks and Open Problems}

The reader should note that we did not use a "binomial" random construction (e.g. keep each edge of $D_{k, \ell}$ with probability $p$ independently) because such a model lacks the concentration we need here. Indeed, for example Janson ([3]) showed that the number of perfect matchings in $G(n, p)$ is not concentrated even when it is quite large, while the number of perfect matchings of $G(n, m)$ is concentrated. We tried to use a binomial random construction and found that the second moments were too large, which in light of Janson's result makes sense (for example derangements in our graph are just a union of several perfect matchings on bipartite graphs).

There are still interesting open problems in [1]. In particular it is still open whether $S$, the set of possible ratios $(d / p)_{G}$, is equal to $\mathbb{Q} \cap[0,1 / 2]$. Here we would like to pose another open problem that is mostly unrelated to our result. In particular, we ask about stability for digraphs whose ratio $(d / p)_{G}$ is close to $1 / 2$ : is it possible that, if a graph has $(d / p)$ in the interval $[1 / 2-c, 1 / 2]$ for sufficiently small $c$, then it must be "nearly" a blowup of a $t$-cycle with $t>t(c)$ ? 


\section{References}

[1] M. Bucic, P. Devlin, M. Hendon, D. Horne, and B. Lund. Perfect matchings and derangements on graphs. J. Graph Theory, 97(2):340-354, 2021.

[2] A. Frieze and M. Karoński. Introduction to random graphs. Cambridge University Press, Cambridge, 2016.

[3] S. Janson. The numbers of spanning trees, Hamilton cycles and perfect matchings in a random graph. Combin. Probab. Comput., 3(1):97-126, 1994. 